\title{
A!
}

This is an electronic reprint of the original article.

This reprint may differ from the original in pagination and typographic detail.

Azangoo, Mohammad; Taherkordi, Amir; Olaf Blech, Jan

\section{Digital Twins for Manufacturing Using UML and Behavioral Specifications}

\section{Published in:}

Proceedings of the 25th IEEE International Conference on Emerging Technologies and Factory Automation, ETFA 2020

DOI:

10.1109/ETFA46521.2020.9212165

Published: 01/09/2020

Document Version

Peer reviewed version

Please cite the original version:

Azangoo, M., Taherkordi, A., \& Olaf Blech, J. (2020). Digital Twins for Manufacturing Using UML and Behavioral Specifications. In Proceedings of the 25th IEEE International Conference on Emerging Technologies and Factory Automation, ETFA 2020 (pp. 1035-1038). [9212165] (Proceedings IEEE International Conference on Emerging Technologies and Factory Automation). IEEE. https://doi.org/10.1109/ETFA46521.2020.9212165

This material is protected by copyright and other intellectual property rights, and duplication or sale of all or part of any of the repository collections is not permitted, except that material may be duplicated by you for your research use or educational purposes in electronic or print form. You must obtain permission for any other use. Electronic or print copies may not be offered, whether for sale or otherwise to anyone who is not an authorised user. 
(C) 2020 IEEE. This is the author's version of an article that has been published by IEEE. Personal use of this material is permitted. Permission from IEEE must be obtained for all other uses, in any current or future media, including reprinting/republishing this material for advertising or promotional purposes, creating new collective works, for resale or redistribution to servers or lists, or reuse of any copyrighted component of this work in other works. 


\section{Digital Twins for Manufacturing Using UML and Behavioral Specifications}

\author{
Mohammad Azangoo \\ Dep. of Electrical Eng. and Automation \\ Aalto University \\ Espoo, Finland \\ mohammad.azangoo@aalto.fi
}

\author{
Amir Taherkordi \\ Department of Informatics \\ University of Oslo \\ Oslo, Norway \\ amirhost@ifi.uio.no
}

\author{
Jan Olaf Blech \\ Dep. of Electrical Eng. and Automation \\ Aalto University \\ Espoo, Finland \\ jan.blech@aalto.fi
}

\begin{abstract}
Using digital twins, physical manufacturing objects can be virtualized and represented as digital models which are seamlessly integrated in both the physical and the digital space. This allows to simulate, verify and optimize production systems, from the logistical aspects to the manufacturing process and the involved components. A key challenge, in this area, is how to describe digital twins in complex manufacturing systems such that all physical details, processes, and verification needs are modeled at an appropriate and efficient abstraction level, e.g., modeling and detecting divers faults in production processes. To address this challenge, in this paper we present our work on modeling digital twins of manufacturing facilities using UML. UML class diagrams are used to describe static dependencies between entities, as well as to monitor and analyze the dynamic verification and quality aspects of manufacturing such as fault detection and consistency checks. Utilizing the key relevant features of UML in our approach, the designed class diagrams are used and enriched with behavioral models serving as digital twins which can be updated by live data from the manufacturing plant. We present a small example based on simulation programs and a demonstrator. The presented modeling approach and example provide useful insights to UML-based design of digital twins in complex manufacturing systems.
\end{abstract}

Index Terms-UML, digital twin, industry 4.0, automation, simulation model, RFID, OPC UA, factory of the future.

\section{INTRODUCTION}

Digital twins have gained popularity in the context of trends such as Industry 4.0 [1] and smart manufacturing [2]. It is typically assumed that a digital twin is a digital counterpart of a (usually) physical entity. Furthermore, we expect some form of link between the entity and the digital copy so that information about the state of the entity is used to update the digital copy. Keeping track of objects in a factory is an important application of digital twins. The status of the digital twin can be updated once new information is made available on the current status of the physical counterpart. This information is gathered by sensors. If no recent information is available, we can use simulation to estimate the current status. This can be based on past information and the general characteristics of the monitored object.

The complexity of manufacturing processes as well as the size of typical plants raise a number of challenges on the management and maintenance in such systems which can be tackled by digital twins [3]. First, manufacturers need to manage and control every step of the processes in a more efficient manner and make necessary reconfigurations while taking all the variables into account. Second, it may be needed to capture the location and condition of every asset in the factory which becomes more challenging in the case of big and complex production processes and high quantity of the assets to be tracked. Beyond these, manufacturers need to have real-time information about possible faults and the causes, predict the performance, analyze every maintenance issue and bottlenecks for multiple possible outcomes, etc. Digital twins can address the above issues by controlling and managing assets and complete processes. However, to efficiently leverage the features of digital twins, it is crucial to adopt a right modeling approach for manufacturing systems which are often big with complex production processes.

In this paper, we present a UML-based framework for modeling digital twins and describing the connection to the real system. The proposed framework is characterized by an efficient digital twin modeling approach that can capture different static and dynamic complexities of smart manufacturing systems in a manageable manner. Considering the former, the physical objects, their properties and their relations are modeled using UML class diagrams with appropriate abstraction levels for divers digital twin classes in manufacturing systems. The latter is addressed through generic behavioural models that describe different monitoring requirements, from dynamic verification to quality checks in manufacturing processes. We present an implementation in which a digital twin can receive data from a real system, but also has some simulation capabilities to estimate the state in the time where no updates are available. Our implementation is based on our Aalto Factory of the Future ${ }^{1}$ infrastructure.

The rest of the paper is organized as follows. In Section II, we discuss related work, while our approach to digital twins in manufacturing systems is introduced in Section III. The concluding remarks are given in Section IV.

\section{RELATED WORK}

Digital twins can be modeled at different levels of details based on the complexity of the target system, including predigital twin, digital, adaptive digital twin, and intelligent digital twin [4]. A variety of ways to specify digital twins have been investigated ranging from CAD models to enriched maintenance protocols. Standards such as AutomationML [5] can be used to describe system setups and thereby serve as an ingredient for a digital twin.

\footnotetext{
${ }^{1}$ https://www.aalto.fi/en/futurefactory
} 


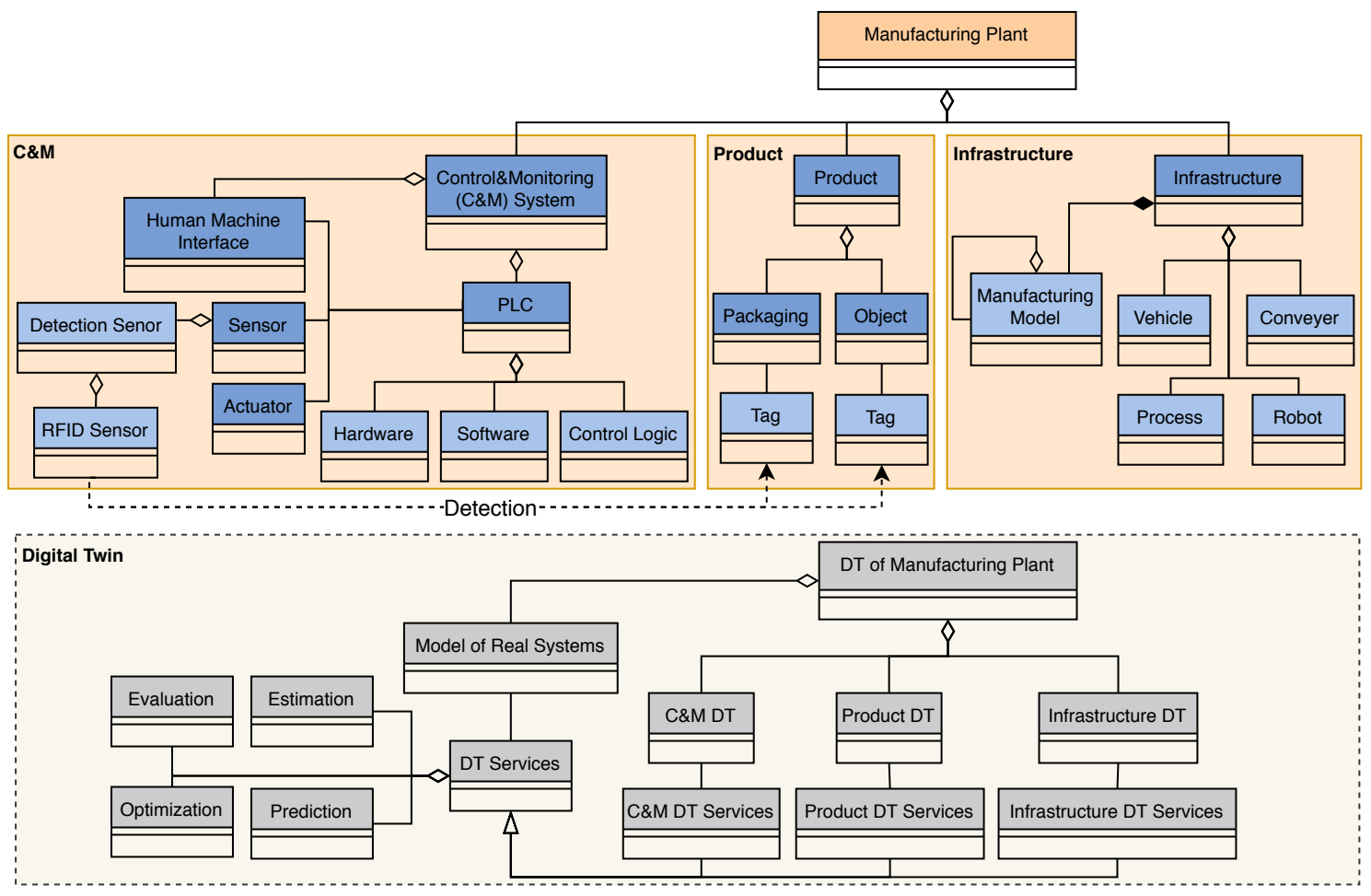

Fig. 1. Abstracted overview of UML class diagram for manufacturing systems and their digital twins

There are basically two main categories of digital twins: data-driven digital twins and model-based digital twins [6]. Data driven digital twins are seen as a manifestation of both known and unknown parts of the system [7]. The model-based digital twin is aimed to represent dynamic and static behavior of the system based on its known physical characteristics, in addition to reducing the risk of management and operation by considering more number of uncertainties [6]. Model based digital twin need to connect the (usually) physical models of subsystems which could be very challenging in complex and multidisciplinary systems, e.g., [8] presents three different types of models for product, process and operation in manufacturing systems, in addition to an integration procedure to make generic digital twins for the whole system is presented. In that paper, Model Based Definition (MBD) technology with geometric information is used for products modeling, as well as for demonstrating multivariate modeling of the process. The UML class diagram has been used to show the relation between the key elements of the process, likewise several forms of modelings such as Petri nets can be used for different operational aspects.

In [9], a new concept is proposed to derive the digital twin model from a digital product description to automatically perform assembly planning and orchestrate the production resources in a manufacturing cell. The authors leverage UML to model the entities of manufacturing cells. A similar implementation is described in [10]. In [11], a UML-based digital twin modeling method for a $\mathrm{CNC}$ machine tool is proposed. This method encompasses a multi-domain unified modeling method, a mapping method and an autonomous strategy.
In [12], the initial concepts for the UML based modeling of the RFID-enabled flexible manufacturing systems are proposed, where it is claimed that using UML can make re-design and also development of the system more efficient.

Digital twins and related concepts are key enablers for industry digitization, but in different disciplines and also in the literature there is no common understanding about this concept [3]. In this work, we bring UML and behavioral models together to present a generic solution for digital twin modeling in discrete manufacturing.

\section{Digital Twins for Manufacturing Systems}

Our approach is based on the UML class diagram to model digital twins and their relation to the physical counterparts. The principal concept is exemplified in Figure 1. The main elements of any manufacturing system are mechanical, chemical and electrical infrastructure, control and monitoring system (automation), and products. Digital twins can support added value services like estimation, validation, simulation and learning. In addition, the end users can be in contact with both the real system and its digital twin for achieving more efficiency in the system life cycle. More detailed sub classes, attributes and operations have not been added in Figure 1 for the sake of simplicity. The complexity of digital twins depends on the number of services in the DT Services class. We leverage class inheritance to describe generalization and specialization when talking about entities (e.g., class actuator and a more specialized class gripper), and aggregation to group entities and describe their relationships (e.g., the connection of an RFID reader to a conveyor belt segment as aggregation). 


\section{A. The Physical Setup}

Our EnAS demonstrator is a laboratory scale agile manufacturing prototype which features Festo components. This demonstrator consists of a conveyor belt ring, actuators, sensors, mobile robots and also a distributed wireless control system. The aim of the system is to transport and handle workpieces based on the defined logic in the distributed control system. The flexibility is achieved by using a Festo Robotino $\mathrm{AGV}$ and additional AGVs produced by other manufacturers. In addition, the demonstrator features RFID readers and RFID tags that are attached to objects in the factory floor to make them traceable. We are particularly looking into tracing workpieces. RFID readers are typically attached to conveyor belt segments, but can also be mounted on robots.

We consider RFID detector as one of the most useful instruments in manufacturing industry to prove the concepts which presented in this paper. The Aalto Factory of the Future has been equipped with the TURK RFID systems. The compact TURCK RFID interfaces exchange data between the RFID head and the controller. Also this module includes an instance of the OPC UA server. Other systems that need information from the OPC UA server, need to have the OPC UA client for communication [13].

\section{B. Digital Twin Realization}

Figure 2 shows the high-level connection between the physical setup and the digital twin. Here we introduce a simple conveyor system as a case study for the manufacturing plant to prove the concepts for realization of digital twin using the UML class diagram. The manufacturing plant has objects as products and the conveyor infrastructure controlled by the automation system. In addition, each conveyor can be equipped with some RFID detectors to detect objects in the manufacturing environment. Furthermore, a digital twin of a manufacturing plant has similar twin classes for objects, conveyor\&automation and RFID detectors. It is clear that the form of relations between classes in the main system and the twin system is the same. In addition, the digital twin has a service class for estimation, consistency check and fault detection which can provide beneficial information for system operation. The only available one directional association relation between the digital twin and the real system comes from the automation system which provides the input information for the digital twin. The main element of the described digital twin for the manufacturing system is DTServices which can implement several added value algorithms or calculation models. These can basically address many complex dynamic processing requirements in manufacturing systems, as mentioned before. The definitions of the offered services for the case study are discussed below. The RFID detectors can detect the objects in specific predefined locations, however they are not suitable for tracking objects like GPS or acceleration sensors. Thus, in the automation system we can see only discrete information about the latest detection. However, in the digital twin by using the LocationEstimate service it is possible to anticipate the probable location of the object. The equation 1 shows how the location of the object can be calculated using the information of the latest detection and also the speed of the conveyor:

$$
L_{e i}=L_{i}+V_{j}\left(t-t_{i}\right)
$$

Where $L_{e i}$ is the estimated location of object $O_{i}, L_{i}$ is the location of the last RFID reader which could detect object $O_{i}$, $V_{j}$ is the speed of the corresponded conveyor $C_{j}, t$ represents the current time and $t_{i}$ is the time of the last detection for object $O_{i}$. Additionally, for verifying the general behavior of the manufacturing system Equation 2 can be used.

$$
C S_{i n}= \begin{cases}1 & \text { legal transition } \\ 0 & \text { illegal transition }\end{cases}
$$

Where $C S_{i n}$ is a consistency check function for object $O_{i}$ when it passes the RFID reader No. $n$. If the RFID signals evaluate as a legal transition for the state machine, then only defined transitions in the state transition table can be considered as a legal transition [14]. The next implemented services in digital twin is for fault detection based on delay in transportation introduced in Equation 3.

$$
F D_{i}= \begin{cases}1 & L_{e i}-L_{i}>D\left(R_{i}\right)+\text { Tolerance } \\ 0 & \text { else }\end{cases}
$$

Where $F D_{i}$ is a fault detection function for the object $O_{i}$, $D\left(R_{i}\right)$ is the distance between the last RFID reader which could detect the object $O_{i}$ and the next expected RFID reader for object $O_{i}$, also Tolerance is the maximum time that the system can wait before activating alarm for fault.

\section{Monitoring Infrastructure}

The software for the simulation model, digital twin and graphical interface has been developed in Python. To communicate with the TURCK OPC UA server module, we defined an OPC UA client for digital twin by using the OPC UA library in Python which was developed based on the IEC 62541 client and server and PyPy [15]. Five RFID heads are connected to two compact TURCK RFID modules implemented in the EnAS demonstrator to track objects equipped with RFID HF tags. Figure 3 shows a screenshot of an example simulation model and its digital twin where the first gray conveyor shows the behavior of the actual system. The second orange conveyor shows the interaction between a normal automation and monitoring system where in the case of any detection, it can show the corresponded RFID reader and tag. It is obvious that the automation system can show only the information based on the actual field detection. The next three red conveyors show the functionalities of the digital twin. DT \#1 shows the estimated location of the objects based on Equation 1. DT \#2 shows the result of the consistency check based on Equation 2. DT \#3 can also find predefined faults in the factory floor based on Equation 3 which in this case considers a delay moreover than 10 seconds as a fault. These features allow better monitoring, fault detection, and maintenance (both reactive and predictive) of the system. Sample recorded video for the operation of the developed digital twin is available in [16].

\section{CONCLUSION}

This paper presented our work on digital twins for manufacturing facilities. UML class diagrams are used to describe 


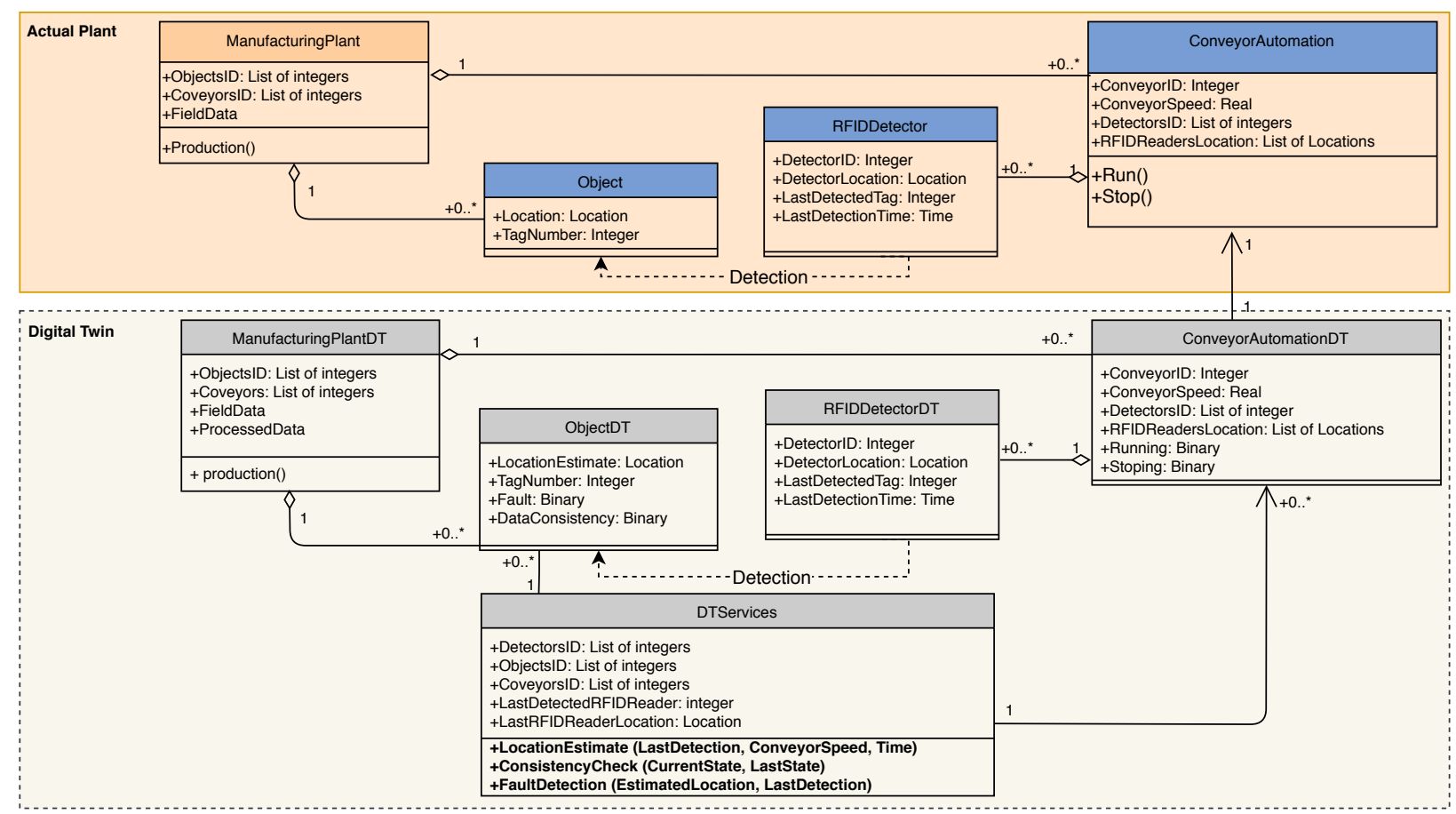

Fig. 2. Developing a UML class diagram for the digital twin of the simple conveyor system

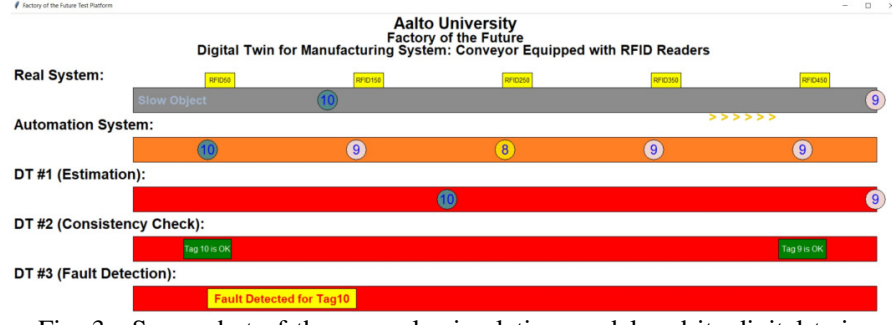

Fig. 3. Screenshot of the example simulation model and its digital twins

digital twins and their connection to physical entities. These descriptions are the basis for additional behavioral descriptions which can be added for simulation purposes and for taking live data from the manufacturing plant into account. In addition, we presented a small example based on simulation programs and a demonstrator where RFID tags are traced in a setup built of a conveyor belt ring and AGV-based transportation services. Future work will extend this scenario and include additional features of AGVs as well as robots. In addition, additional work on distributed digital twins over several devices and architectural layers is of concern to us.

\section{REFERENCES}

[1] H. Kagermann, J.Helbig, A. Hellinger and W. Wahlster, "Umsetzungsempfehlungen für das Zukunftsprojekt Industrie 4.0: Deutschlands Zukunft als Produktionsstandort sichern; Abschlussbericht des Arbeitskreises Industrie 4.0," (In German), Forschungsunion, 2013.

[2] H. S. Kang, J. Y. Lee, S. Choi, H, Kim, J. H. Park, J. Y. Son, ... \& S. Do Noh,"Smart manufacturing: Past research, present findings, and future directions," International journal of precision engineering and manufacturing-green technology, 3(1), 111-128,2016.

[3] W. Kritzinger, M. Karner, G. Traar, J. Henjes and W. Sihn, "Digital Twin in manufacturing: A categorical literature review and classification," IFAC-PapersOnLine, Volume 51, Issue 11, 2018.
[4] A. M. Madni, C. C. Madni and S. D. Lucero, "Leveraging Digital Twin Technology in Model-Based Systems Engineering," Special Issue Model-Based Systems Engineering, 7, 7, 2019.

[5] Drath, R., Luder, A., Peschke, J., \& Hundt, L. (2008, September). AutomationML-the glue for seamless automation engineering. In 2008 IEEE International Conference on Emerging Technologies and Factory Automation (pp. 616-623). IEEE.

[6] F. Jaensch, A. Csiszar, C. Scheifele and A. Verl, "Digital Twins of Manufacturing Systems as a Base for Machine Learning," 2018 25th International Conference on Mechatronics and Machine Vision in Practice (M2VIP), Stuttgart, 2018, pp. 1-6.

[7] A. Rasheed, O. San and T. Kvamsdal, "Digital Twin: Values, Challenges and Enablers From a Modeling Perspective," in IEEE Access, vol. 8, pp. 21980-22012, 2020.

[8] J. Bao, D. Guo, J. Li and J. Zhang, "The modelling and operations for the digital twin in the context of manufacturing," Enterprise Information Systems, V. 13, number4, PP. 534-556, 2019.

[9] Sierla, Seppo, Ville Kyrki, Pekka Aarnio, and Valeriy Vyatkin. "Automatic assembly planning based on digital product descriptions." Computers in Industry 97 (2018): 34-46.

[10] V. Kuliaev, U.D. Atmojo, S. Sierla, J.O. Blech, V. Vyatkin, "Towards Product Centric Manufacturing: From Digital Twins to Product Assembly", 17th International Conference on Industrial Informatics (IEEEINDIN 2019) July 22-25, 2019 Helsinki, Finland.

[11] W. Luo, T. Hu, W. Zhu and F. Tao, "Digital twin modeling method for CNC machine tool," 2018 IEEE 15th International Conference on Networking, Sensing and Control (ICNSC), Zhuhai, 2018, pp. 1-4.

[12] A. Vatankhah Barenji, R. Vatankhah Barenji and M. Hashemipour, "Structural Modeling of a RFID-enabled Reconfigurable Architecture for a Flexible Manufacturing System," Smart SysTech 2013; European Conference on Smart Objects, Systems and Technologies, Erlangen/Nuremberg, Germany, 2013, pp. 1-10.

[13] Turck, "BL ident ${ }^{\circledR}$ RFID System Planning and Engineering, User Manual," Oct. 2017.

[14] M. Azangoo, J. O. Blech and U. D. Atmojo, "Towards Formal Monitoring of Workpieces in Agile Manufacturing," 2020 IEEE International Conference on Industrial Technology (ICIT), Buenos Aires, Argentina, 2020, pp. 334-339, doi: 10.1109/ICIT45562.2020.9067188.

[15] O. Roulet-Dubonnet, "Python OPC-UA Documentation," release 1.0, 2018.

[16] Aalto Factory of the Future. "Digital twin for EnAS Manufacturing Demonstrationr," YouTube, May. 08, 2020 [Video file]. Available: https://www.youtube.com/watch?v=moQ8JHRDoJg. 